\title{
Supporting Information: Overgrowth of Silver Nanodisks on a Substrate into Vertically Aligned Nanopillars for Chromatic Light Polarization
}

\author{
Mahmoud A. Mahmoud* \\ Laser Dynamics Laboratory, School of Chemistry and Biochemistry, Georgia Institute of Technology, \\ Atlanta, Georgia 30332-0400 \\ *E-mail: mmahmoud@gatech.edu
}



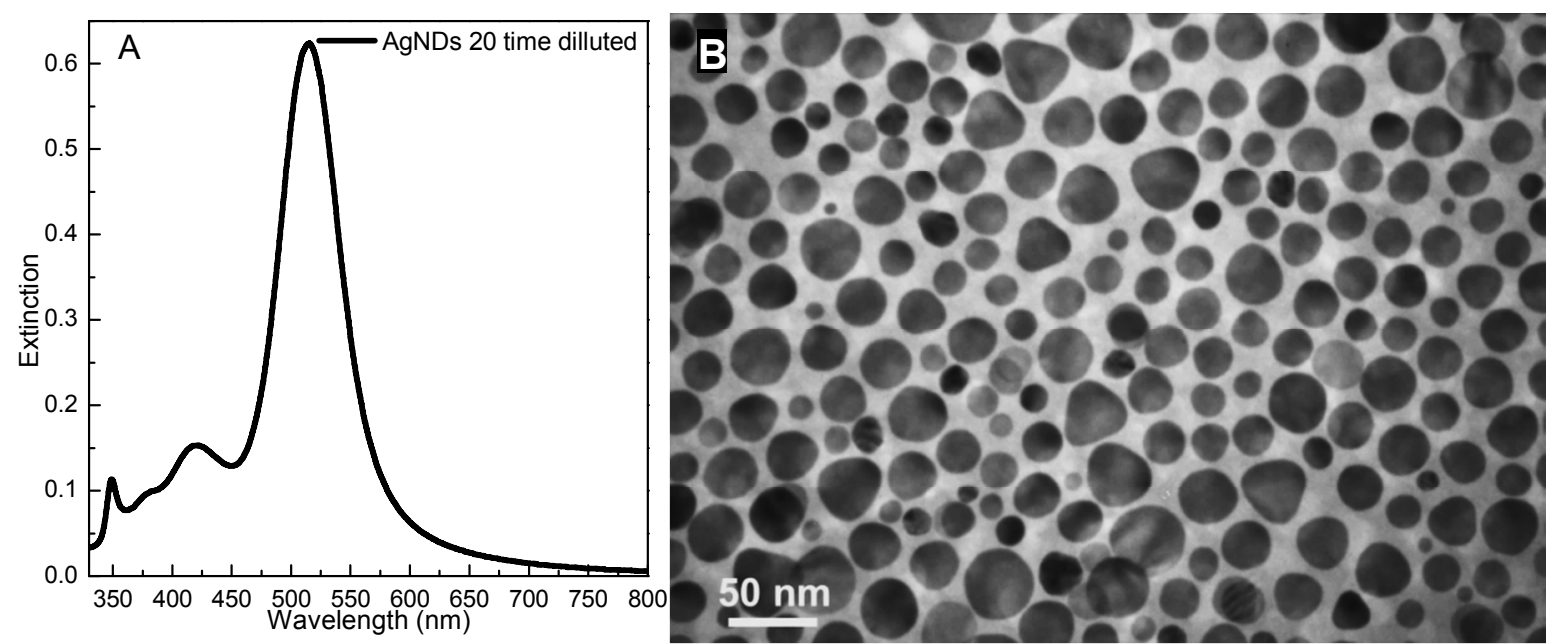

Figure S1 A) The LSPR extinction spectrum of the AgNDs in ethanol-chloroform mixture after 20 time dilution with ethanol. B) TEM image of AgNDs of $26.8 \pm 6.1 \mathrm{~nm}$ diameter and $8.3 \pm 1.1$ nm thickness.

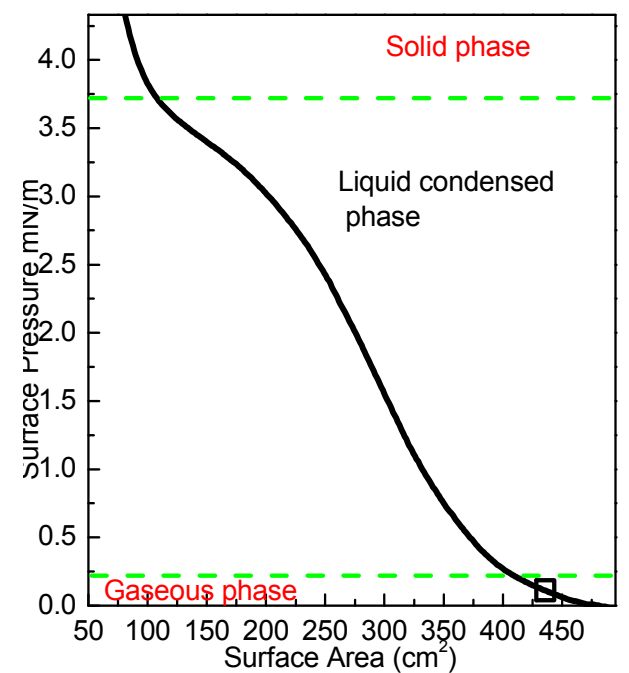

Figure S2 LB isotherm of AgNDs of diameter of $26.8 \pm 6.1 \mathrm{~nm}$ and thickness of $8.3 \pm 1.1 \mathrm{~nm}$. The isotherm showed three different phases as in case of regular isotherms. 


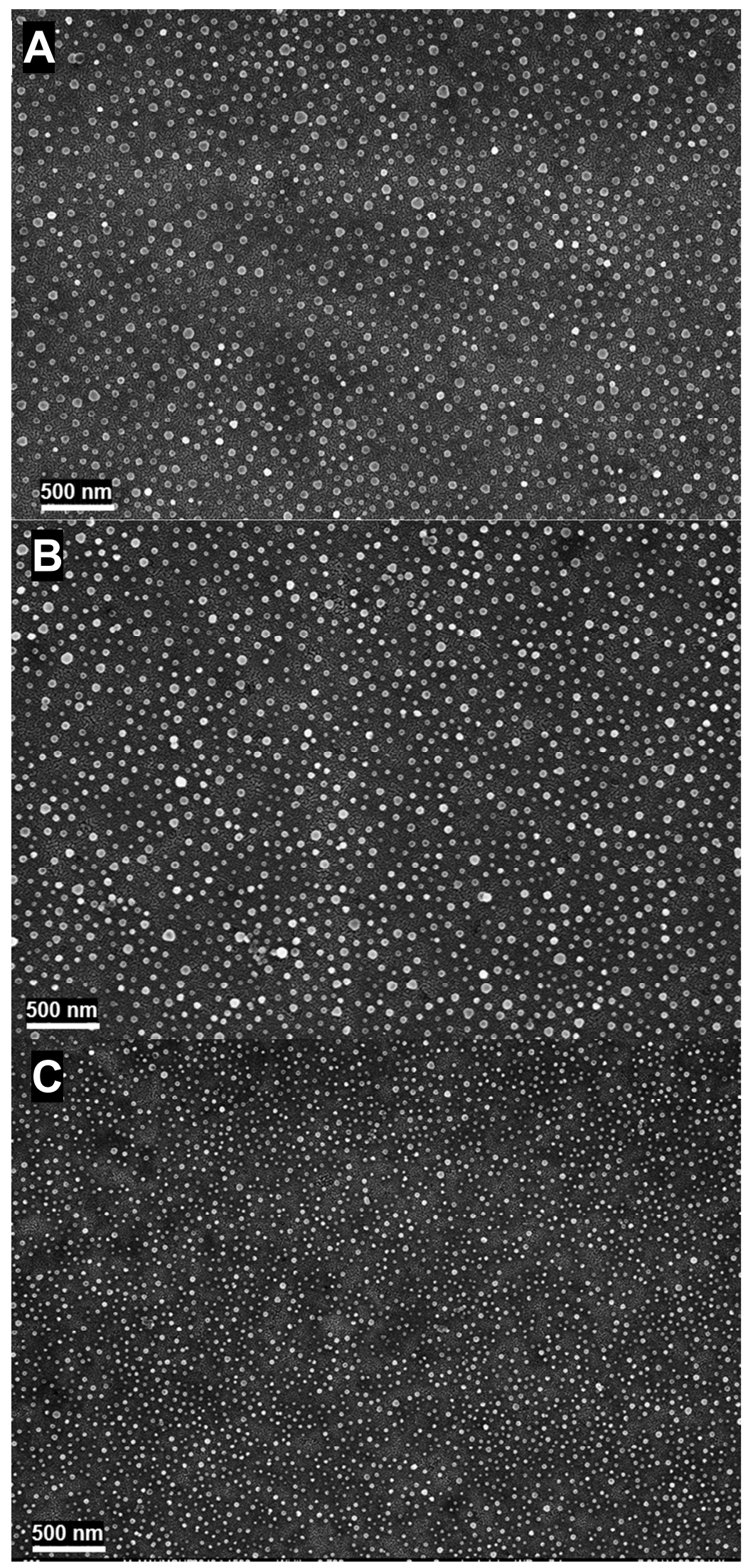

Figure S3 Low magnification SEM image of: A) bigger hexagonal AgNDs of $55.5 \pm 5.9$ $\mathrm{nm}$ diameter and $23.6 \pm 4.2 \mathrm{~nm}$ thickness resulted from the symmetrical overgrowth of the AgNDs monolayer on quartz substrate. B) Short AgNPLs of $43.9 \pm 7.2 \mathrm{~nm}$ diameter and $36.2 \pm 4.1 \mathrm{~nm}$ height, which obtained from the overgrowth of the AgNDs LB film by the reduction of silver ions using 78 $\mathrm{mM}$ ascorbic acid in the presence of chloride ions. C) Tall AgNPLs of diameter $32.5 \pm 4.8 \mathrm{~nm}$ of and height of $67.8 \pm 6.9 \mathrm{~nm}$ formed when the concentration of the ascorbic was decreased to be 7.8 $\mathrm{mM}$ in the presence of chloride ions. 

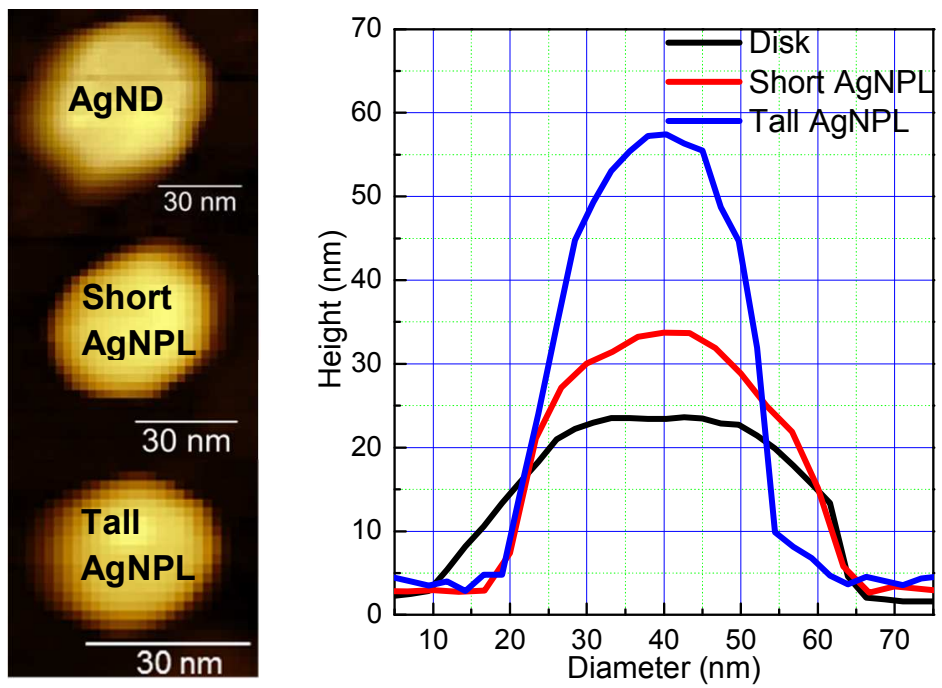

Figure S4 Topographical cross sections along the center of a hexagonal AgNDs obtained from the overgrowth of AgND in the absence of $\mathrm{Cl}^{-}$and AgNPL resulted from the overgrowth of AgNDs using 7.8 (blue) and 78 (red) mM AA.
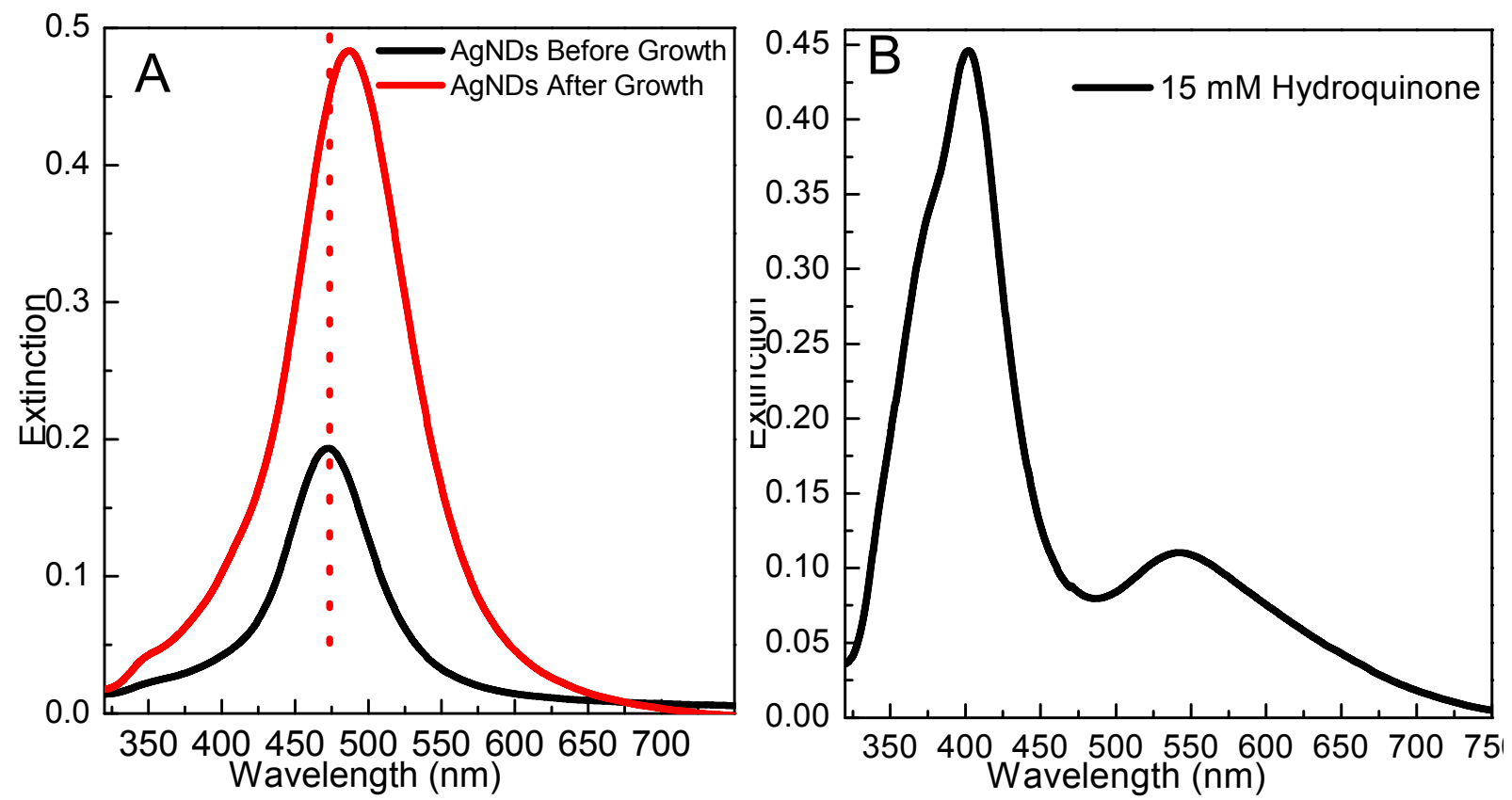

Figure S5 LSPR spectrum of the AgNDs: A) before (black spectrum) and after the overgrowth in the absence of $\mathrm{Cl}^{-}$. The LSPR spectrum was red shifted by $8 \mathrm{~nm}$ and the intensity was increased after the overgrowth. B) LSPR spectrum of AgNPLs prepared by the overgrowth of AgNDs using $15 \mathrm{mM}$ of hydroquinone. The longitudinal and the transverse plasmon modes appeared at 542 and $405 \mathrm{~nm}$, respectively. 

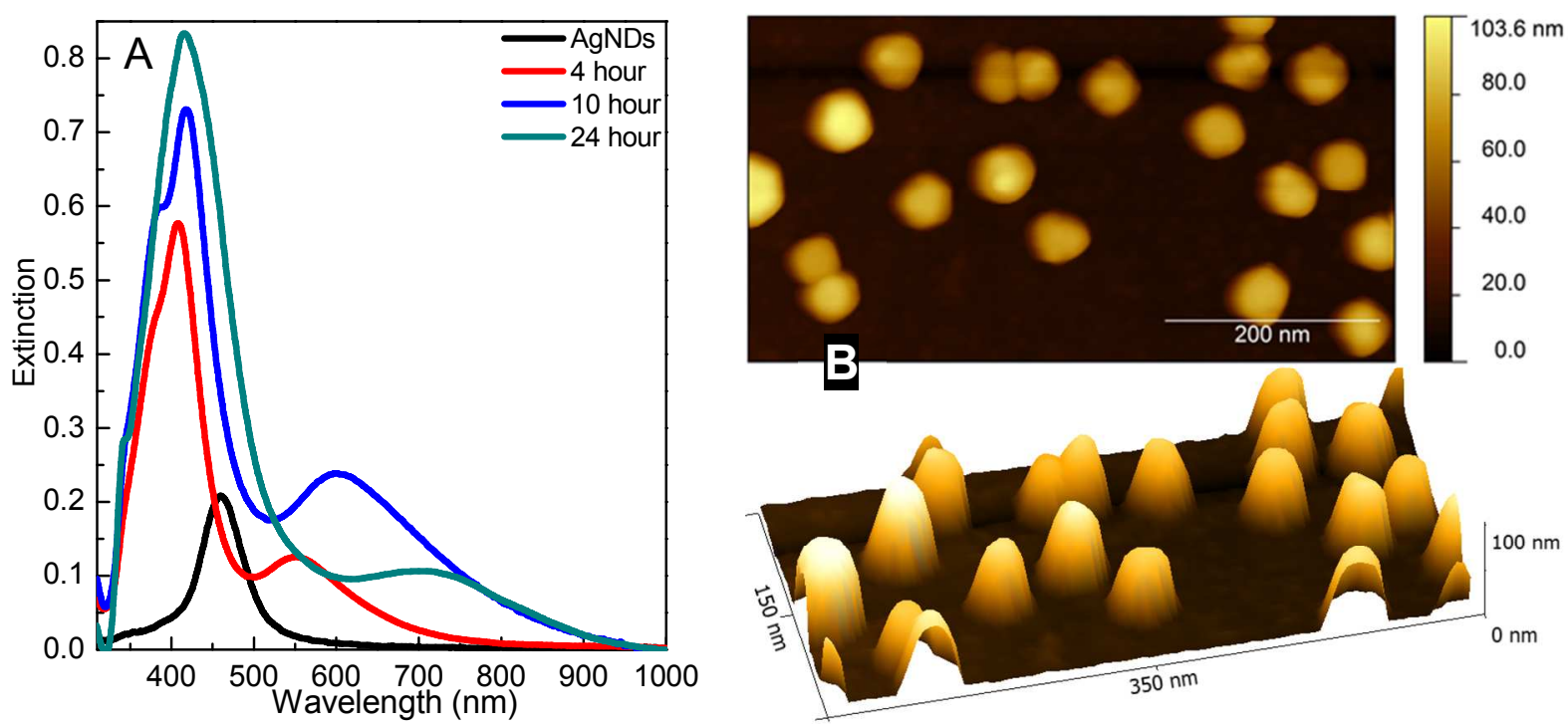

Figure S6 A) LSPR spectrum of the AgNPLs resulted from the overgrowth AgNDs using $7.8 \mathrm{~nm}$ AA collected at growth time of 4, 10, and 24 hours. B) AFM image the AgNPLs fabricated from the 24 hours overgrowth of AgNDs using $7.8 \mathrm{~nm}$ AA.
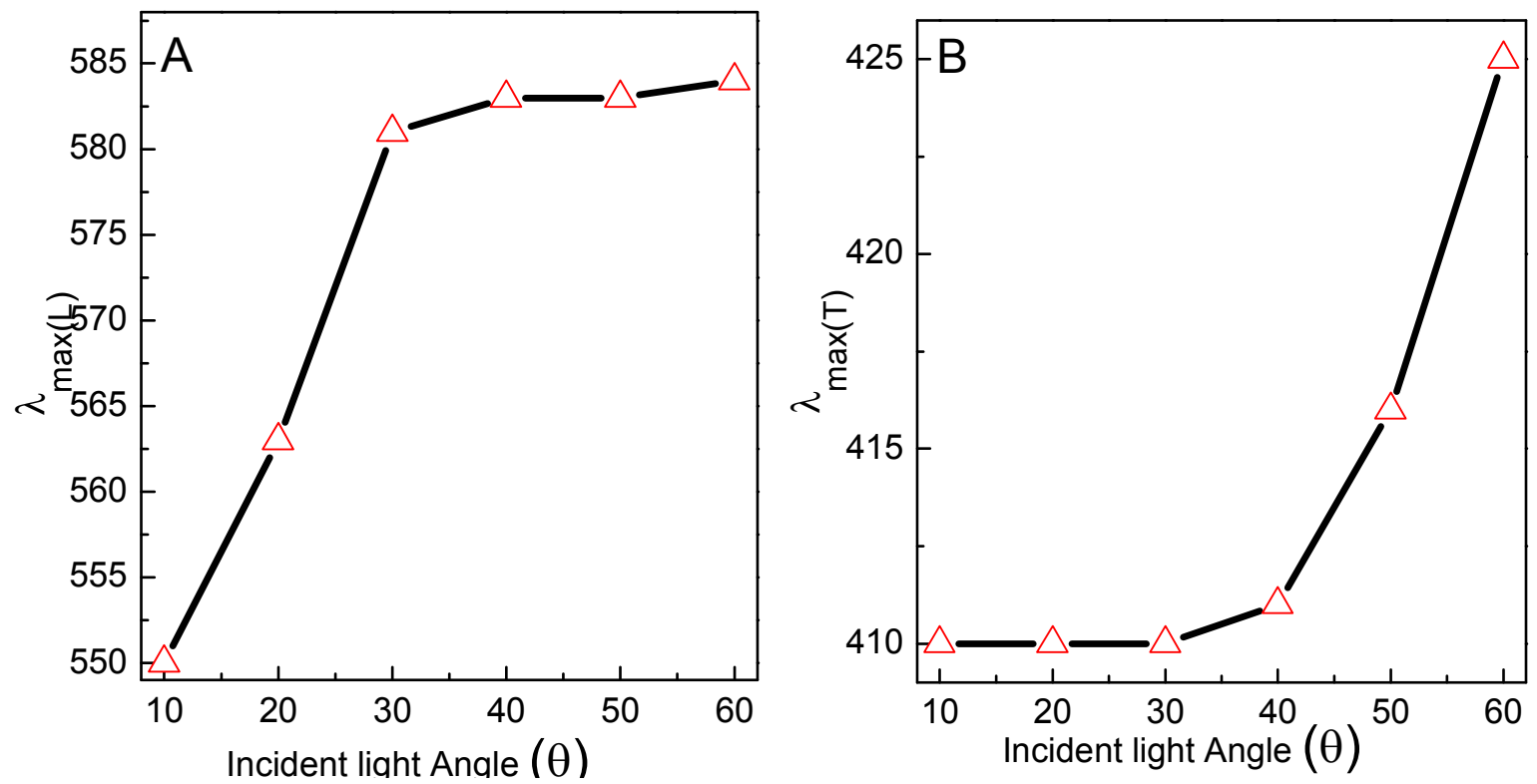

Figure S7 The relationship between: A) the longitudinal LSPR peak position of the AgNPLs and the angle of excitation. B) The transverse LSPR peak position and the angle of excitation. 


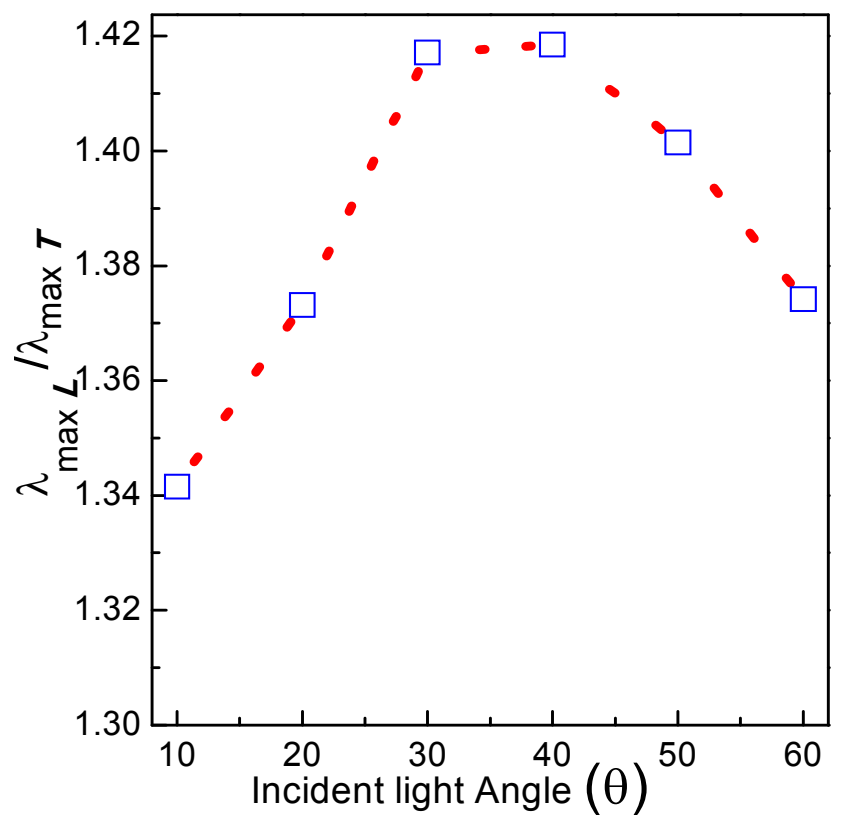

Figure S8 shows the relationship between the angle of excitation $\varphi$ and the ratio between the LSPR maximum peak position of the longitudinal and transverse mode. 